\title{
CAPACIDADE FUNCIONAL DE IDOSOS DE UMA COMUNIDADE RURAL DO RIO GRANDE DO SUL ${ }^{a}$
}

\author{
Ilva Inês RIG O ${ }^{b}$, Lisiane M anganelli G irardi PASKULINcc, Eliane Pinheiro de M ORAIS
}

\section{RESUMO}

Estudo seccional realizado com 34 pessoas idosas de uma comunidade rural do Rio G rande do Sul, em 2008, com o objetivo de analisar sua capacidade funcional e comparar as variáveis estudadas entre sexos. As variáveis sociais, econômicas, demográficas, relacionadas à saúde/ doença, além do M iniexame do Estado M ental (M EE M ) e da Escala de A tividades da Vida Diária (AVD) do Older American Resources and Services (OARS), foram coletadas através de inquérito domiciliar. A análise dos dados foi realizada por meio de estatística descritiva. Em relação aos homens, as mulheres tinham melhor escolaridade, participavam menos das atividades comunitárias, tinham escores semel hantes no MEEM , pior percepção de saúde e maior dependência nas AVD. Destacamse as singularidades das pessoas idosas do meio rural e a necessidade de desenvolver ações que promovam a sua capacidade de adaptação nesse contexto, com o objetivo de minimizar as incapacidades.

D escritores: Saúde do idoso. A tividades cotidianas. População rural.

\section{RESUMEN}

E studio transversal realizado con 34 ancianos de una comunidad rural de Rio G rande do Sul, B rasil, en 2008, con el objetivo de analizar su capacidad funcional y comparar las variables entre sexos. L as variables sociales, económicas, demográficas, relacionadas con la salud y la enfermedad, el M ini-M ental State Examination (M M SE) y la E scala de Actividades de $L$ a Vida D iaria (A VD) de Older A merican R esources and Services (OARS) han sido recogidos mediante encuesta de hogares. E I análisis de los datos se realizó mediante estadística descriptiva. E n comparación con los hombres, las mujeres estaban mejor educadas, participaban menos en las actividades comunitarias, tenían puntuaciones similares en el M M SE, peor percepción de la salud y mayor dependencia en las A VD. Se destacan las singularidades de las personas mayores en las zonas rurales y la necesidad de desarrollar acciones que promuevan su capacidad de adaptación en este contexto, con el objetivo de minimizar la discapacidad.

D escriptores: Salud del anciano. Actividades cotidianas. Población rural.

$T$ ítulo: Capacidad funcional de los ancianos de una comunidad rural de $\mathrm{R}$ io $\mathrm{G}$ rande do $\mathrm{Sul}$.

\section{ABST RACT}

Cross-sectional study, conducted with 34 elder people from a rural community of Rio G rande do Sul , B razil, in 2008. The objective is to evaluate their functional capacity and to compare inter est variables betw een sexes. The household survey addressed social, economic and demographic variables, aspects related to health / disease, the M ini-M ental StateE xamination (M M SE ) and the $S$ cale of Activities of $D$ aily $L$ iving (ADL ) of the Older A merican R esources and Services (OARS). D escriptive statistics w ere used for data analysis. Compared to men, women were more likely to be more educated, have less community activities, have similar M E E M scores, worst health perception and were more dependent to perform AD L. T he peculiarities of rural elder persons are highlighted, as well the need to tailor actions to promote their adaptability in this context and allow the minimization of disabilities.

Descriptors: $\mathrm{H}$ ealth of the elderly. Activities of daily living. Rural population.

$T$ itle: $\mathrm{F}$ unctional capacity of elder people from a rural community of Rio $\mathrm{G}$ rande do Sul.

\footnotetext{
${ }^{\text {aA }}$ rtigo originado do T rabal ho de Conclusão de Curso apresentado em 2008 na Escola de Enfermagem da Universidade Federal do Rio Grande do Sul (EEUFRGS).

${ }^{b}$ Enfermeira graduada pela EEUFRGS, Porto Alegre, Rio Grande do Sul, Brasil.

'D outora em Ciências, Professora Adjunta da EEUFRG S e Professora Permanente do Programa de Pós-G raduação da EEUFRGS, Porto Alegre, Rio Grande do Sul, Brasil.

${ }^{d}$ D outora em Enfermagem F undamental, Professora A djunta da E EU F RG S e Professora Colaboradora do Programa de Pós-G raduação da EEUFRGS, Porto Alegre, Rio Grande do Sul, Brasil.
} 


\section{INT RODUÇÃO}

0 envelhecimento populacional está relacionado à diminuição das taxas de fecundidade e de natalidade e aos esforços em melhorar as condições de vida da população. $N$ as sociedades em desenvolvimento, isso ocorre de modo aceler ado, sem haver um planejamento para responder às necessidades dos idosos, acentuando as desigualdades e aumentando a demanda por serviços de saúde ${ }^{(1)}$. Estudos epidemiológicos vêm sendo realizados a fim de identificar essas necessidades, principalmente nas zonas urbanas da Região Sudeste do Brasil $^{(1-3)}$, e pouco ainda tem sido divulgado sobre 0 perfil dos idosos de áreas rurais no contexto nacional ${ }^{(4-6)}$.

A capacidade funcional é uma das formas mais adequadas para avaliar as condições dos idosos, pois traduz um conceito ampliado de saúde, entendido como a existência de habilidades físicas e mentais para a manutenção da autonomia e da independência, envolvendo múltiplos aspectos da vida do idoso, como condições socioeconômicas, cognitivas e de saúde, entre outros ${ }^{(3)}$.

U m dos aspectos importantes na avaliação da capacidade funcional é a capacidade para realização das Atividades da Vida Diária (AVDs), que são compostas pelas A tividades Físicas e Instrumentais da Vida Diária (AFVDs e AIVDs). As AF VD s são as cotidianas, que quantificam a capacidade de autocuidado do idoso. Já as AIVDS são atividades mais complexas, que indicam o grau de independência do idoso nas atividades comunitárias $^{(7)}$.

Considerando que a capacidade funcional do idoso envolve uma gama de aspectos de sua vida, foram objetivos deste estudo: identificar a capacidade funcional de idosos residentes em uma comunidade rural do Rio Grande do Sul, considerando suas características sociodemográficas de saúde/ doença e as condições cognitivas e funcionais; comparar as variáveis citadas ponderando o sexo dos idosos em questão.

A investigação foi realizada no município de N ova Roma do Sul, Rio Grande do Sul. D e acordo com dados coletados no Censo de 2000, 16,5\% da população do município era composta por idosos, enquanto essa proporção para o país era de $8,6 \%{ }^{(8)}$, indicando um processo de franco envelhecimento populacional. Em virtude disso, propõe-se a rea- lização deste estudo, a fim de verificar as condições de vida e saúde das pessoas idosas que lá residem.

D entre as contribuições para a enfermagem, trazidas pelo estudo, destaca-se a identificação das necessidades de cuidado desses idosos, disponibilizando infor mações que auxiliarão no planejamento e proposição de ações adequadas às características dessa população, além de fornecer subsídios para a caracterização da população idosa rural brasileira.

\section{MÉTODOS}

Estudo seccional realizado através de inquérito domiciliar numa comunidade rural do município de N ova Roma do Sul, Rio Grande do Sul, Brasil. 0 município localiza-se na região nordeste do estado do Rio G rande do Sul. Sua população é composta, basicamente, por descendentes de imigrantes italianos. D os 3032 habitantes, 500 eram ido$\operatorname{sos}^{(8)}$ e 39, residentes na comunidade selecionada, conforme informações da associação de moradores do local.

0 inquérito domiciliar foi operacionalizado com o auxílio do mapa da região e foram visitadas as 35 residências pertencentes à área de abrangência da comunidade. $N$ os casos em que os moradores não foram encontrados, a pesquisadora e coletadora dos dados fez outras duas tentativas de contato. Os critérios de inclusão no estudo foram: ter 60 anos ou mais e residir na comunidade. Foi previsto também que idosos portadores de deficiência auditiva grave não corrigida e/ ou que atingissem escore inferior a 12 no $\mathrm{M}$ iniexame do Estado $\mathrm{M}$ ental ${ }^{(9)}$ teriam as informações coletadas com o auxílio do cuidador principal. Os critérios de exclusão foram não encontrar os moradores/ idosos na residência após três visitas e não aceitar participar do estudo.

A o final, foram identificadas 39 pessoas com 60 anos ou mais e residentes na comunidade, das quais duas não aceitaram participar e três não foram encontradas na residência, após três visitas, totalizando 34 idosos entrevistados. Em apenas um dos casos foi necessário o auxílio do cuidador, pois o idoso possuía deficiência auditiva.

Levando em conta que a capacidade funcional envolve diversos aspectos da vida do idoso, foram estudadas variáveis demográficas e socioeconômicas, relacionadas à saúde-doença, ao estado 
cognitivo e à capacidade para realização de atividades de vida diária. As variáveis socioeconômicas e demográficas investigadas foram: idade, sexo, escolaridade, situação ocupacional, estado conjugal arranjo domiciliar, interação social. Já as variáveis relacionadas à saúde-doença er am a autoaval iação de saúde, as morbidades referidas e as limitações causadas pelas mesmas.

A avaliação cognitiva ocorreu por meio do $M$ iniexame do Estado M ental (M EEM), traduzido, validado no Brasil( ${ }^{(10)}$ e adaptado para o uso com idosos ${ }^{(9)}$. Para rastrear o comprometimento cognitivo, foram adotados os pontos de corte 13 ou menos, para idosos analfabetos e 18 ou menos, para idosos que sabiam ler e escrever ${ }^{(10)}$, em virtude do tipo de ensino formal oferecido a eles no passado.

Foi utilizada, ainda, a versão brasileira da E scala de AVDs do Older A merican Resources and Services (OARS) traduzida e validada no Brasil(7). $N$ essa escala, a capacidade do idoso para realizar as AFVD e AIVD é classificada em "realiza com ajuda" e "realiza sem ajuda" com o máximo de 28 pontos. A classificação indica: independência para os idosos que não necessitam de ajuda em nenhuma das AVD; dependência leve para os idosos que necessitam de ajuda em uma até três atividades; dependência moderada para os indivíduos que necessitam de ajuda em quatro até seis atividades e dependência grave para os que são incapazes de realizar sete ou mais atividades sem auxílio(7).

A coleta de dados ocorreu nos meses de abril e de maio de 2008. Os dados foram gerenciados com o programa Statistical Package for the Social Sciences (SPSS) 12.0, utilizando a estatística descritiva. Foram comparadas as proporções das variáveis estudadas entre os sexos. No M EE M, optou-se por relacionar também a média dos escores obtidos no teste à escolaridade, pois esta influencia o desempenho no mesmo ${ }^{(10)}$. Os idosos participantes constituíram o universo da comunidade selecionada, portanto foi desnecessário realizar testes de significância estatística para a comparação das proporções entre sexo e as variáveis de interesse.

O projeto foi aprovado no Comitê de Ética em Pesquisa da U niversidade Federal do Rio G rande do Sul (Processo no 2007/ 824) em 14 de março de 2008. T odos os participantes assinaram um T ermo de Consentimento Livre e E sclarecido, ficando uma via com os mesmos e outra com o pesquisador.

\section{RESULTADOS}

Conforme a T abela 1, a maior parte dos entrevistados era do sexo feminino $(55,9 \%)$ e estava na faixa etária de 60 a 69 anos (55,9\%). A média etária dos participantes foi 69,8 anos ( $D P \pm 7,2$ anos). H ouve semelhança entre a média etária dos homens $(69,6$ anos, $D P \pm 7,1)$ e mulheres $(70,0$ anos, DP $\pm 7,5)$. Verificou-se maior proporção de muIheres (36,8\% entre 70 e 79 anos e 10,5\% entre 80 e 89 anos) em relação aos homens (33,3\% e 6,7\% respectivamente) nas faixas etárias mais avançadas.

Quanto à escolaridade, 55,9\% dos respondentes possuíam até 3 anos de estudo. D estaca-se que a proporção de mulheres com escolaridade entre 1 e 7 anos de estudo (89,5\%) foi superior a de homens (73,4\%). Do mesmo modo, o percentual de analfabetismo diferiu entre os sexos, pois 33\% dos homens relataram não saber ler e escrever, enquanto que este percentual era de 5,3\% entre as mulheres. E m relação ao estado conjugal, 58,8\% dos participantes possuíam companheiro. Considerando sexo e estado conjugal, $66,7 \%$ dos homens e $52,6 \%$ das mulheres possuíam companheiro. T odos os entrevistados eram aposentados, preponderando os que continuavam trabal hando $(94,1 \%)$. Quanto à diferença entre sexos, todos os homens mantinham atividade laboral e 10,5\% das idosas já não trabaIhavam.

A atividade social mais citada pelos idosos foram as festas da comunidade, $85,3 \%$ deles participavam sempre ou quase sempre desses eventos. Todos os homens mencionaram participar dessa atividade, sempre ou quase sempre, em contrapartida $73,7 \%$ das mulheres relataram tal frequência. 0 encontro no salão comunitário era a atividade da qual os idosos participavam maciçamente $(97,1 \%)$. Por outro lado, observa-se que $79,4 \%$ dos idosos nunca foram ao grupo de idosos, que ocorre mensalmente na área urbana do município. N esse grupo, a participação das mulheres $(26,4 \%)$ foi superior à participação dos homens (5,3\%).

Verifica-se, ainda, que grande parte dos entrevistados morava em domicílios em que residiam mais de uma ger ação (82,3\%). Com relação ao sexo, observou-se que residir em domicílios com três gerações ou mais foi mais frequente entre as muIheres $(68,5 \%)$ que entre os homens $(60 \%)$. 
T abela 1 - Caracterização sociodemográfica dos idosos segundo sexo ( $N$ =34). N ova Roma do Sul, RS, 2008.

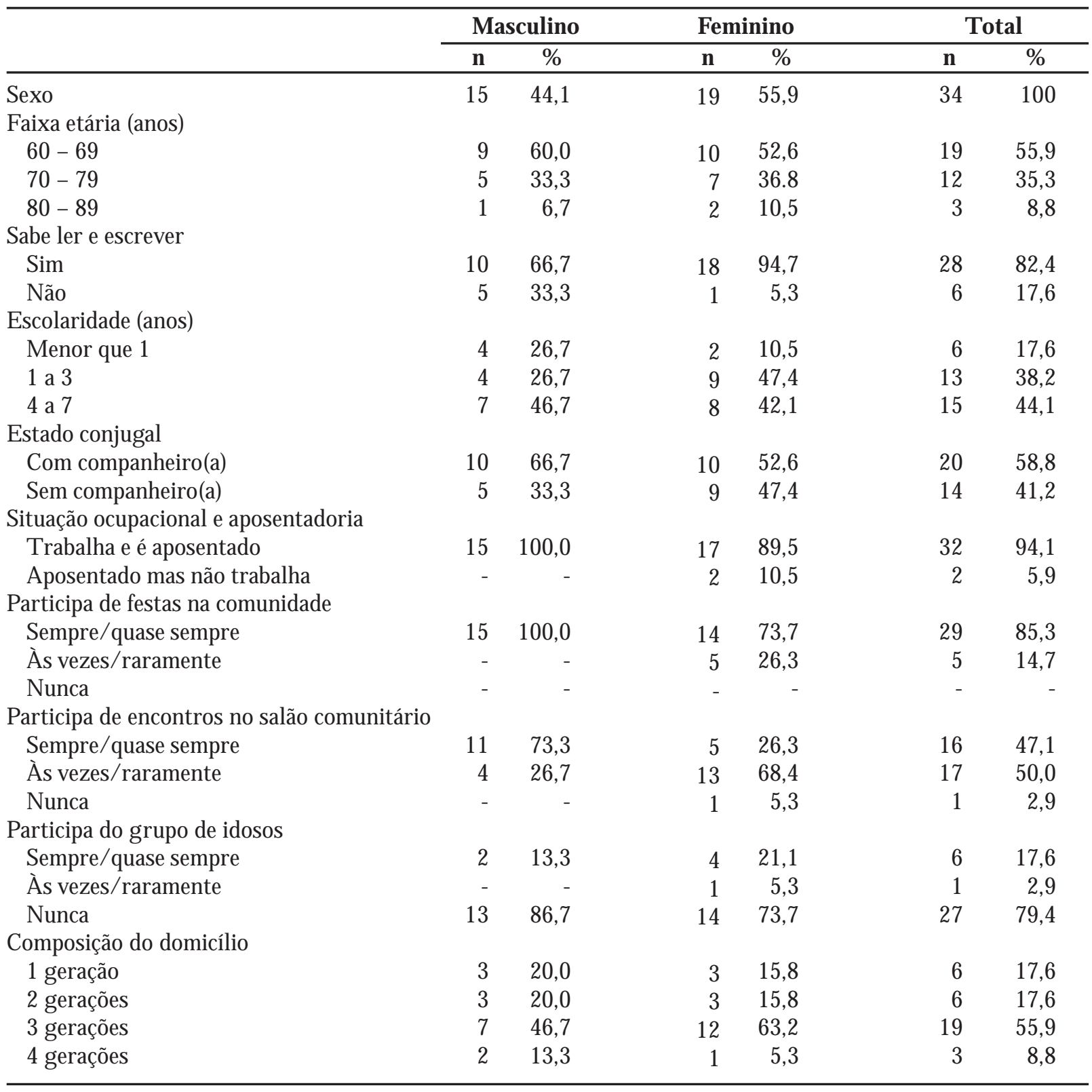

T abela 2 - E scores médios e desvio padrão no M E EM dos idosos, segundo escolaridade e sexo ( $N=33$ ). N ova Roma do Sul, RS, 2008.

\begin{tabular}{lccccc}
\hline & $\mathbf{n}$ & M édia & D P & Mínimo & Máximo \\
\hline M édia geral & 33 & 25,8 & 2,8 & 20 & 30 \\
E scolaridade & 5 & 23,6 & 2,30 & 20 & 26 \\
$\quad$ M enos que 1 ano & 13 & 24,4 & 2,7 & 22 & 30 \\
1 a 3 anos & 15 & 27,7 & 1,6 & 24 & 30 \\
4 a 7 anos & & & & & \\
Sexo & 14 & 26,7 & 2,2 & 23 & 30 \\
M asculino & 19 & 25,1 & 3,0 & 20 & 29 \\
Feminino & & & & & \\
\hline
\end{tabular}

Legenda: D P : desvio padrão. 
A T abela 2 apresenta os escores médios do MEEM segundo sexo e escolaridade. 0 menor valor obtido no teste foi de 20 pontos, o maior, 30 pontos e a média geral, 25,8 ( $D P \pm 2,8$ ). Quando considerado o sexo, os homens obtiveram escores médios ligeiramente superiores aos escores das mulheres (26,7; DP $\pm 2,2$ e 25,1; DP $\pm 3,0$ respectivamente). Em relação à escolaridade e escores obtidos no teste, a média entre os idosos com escolaridade menor que um ano foi de 23,6 ( $D P \pm 2,3$ ). Por outro lado, os idosos que estudaram de um a três anos obtiveram média de 24,4 ( $D P \pm 2,7$ ) e os com escolaridade de quatro a sete anos, 27,7 (DP $\pm 1,6)$. N enhum dos idosos apresentou comprometimento cognitivo, segundo os pontos de corte propostos.

T abela 3 - Auto-avaliação de saúde, saúde comparada aos demais, morbidades referidas e limitação causada e grau de dependência dos idosos, segundo sexo ( $N=34)$. N ova Roma do Sul, RS, 2008.

\begin{tabular}{lrrrrrr}
\hline & \multicolumn{2}{c}{ M asculino } & \multicolumn{2}{c}{ Feminino } & \multicolumn{2}{c}{ T otal } \\
\cline { 2 - 7 } & $\mathbf{n}$ & $\%$ & $\mathbf{n}$ & $\%$ & $\mathbf{n}$ & $\%$ \\
\hline Autoavaliação de saúde & & & & & & \\
$\quad$ Excelente/ muito boa/ boa & 5 & 33,3 & 4 & 21,1 & 9 & 26,5 \\
$\quad$ Regular & 9 & 60,0 & 13 & 68,4 & 22 & 64,7 \\
$\quad$ Má & 1 & 6,7 & 2 & 10,5 & 3 & 8,8 \\
M orbidades referidas & & & & & & \\
$\quad$ Artropatia & 6 & 40,0 & 13 & 68,4 & 19 & 55,9 \\
$\quad$ Sim & 2 & 33,3 & 2 & 15,4 & 4 & 21,1 \\
$\quad$ Limita muito & 3 & 50,0 & 5 & 38,5 & 8 & 42,1 \\
$\quad$ Limita pouco & 1 & 16,7 & 6 & 46,2 & 7 & 36,8 \\
$\quad$ Não limita & 9 & 60,0 & 6 & 31,6 & 15 & 44,1 \\
$\quad$ Não & & & & & & \\
Hipertensão & 5 & 33,3 & 13 & 68,4 & 18 & 52,7 \\
$\quad$ Sim & - & - & 2 & 15,4 & 1 & 5,6 \\
$\quad$ Limita muito & - & - & 5 & 38,5 & 5 & 27,8 \\
$\quad$ Limita pouco & 4 & 80,0 & 7 & 53,8 & 11 & 61,1 \\
$\quad$ Não limita & 1 & 20,0 & - & - & 1 & 5,6 \\
$\quad$ Respondente substituto & 10 & 66,7 & 6 & 31,6 & 16 & 47,1 \\
$\quad$ Não & & & & & & \\
G rau de dependência nas AV D & 6 & 53,5 & 4 & 21,1 & 12 & 35,3 \\
$\quad$ Independência & 6 & 40,0 & 12 & 63,2 & 18 & 52,9 \\
Dependência leve & 1 & 6,7 & 2 & 10,5 & 3 & 8,8 \\
$\quad$ Dependência moderada & - & - & 1 & 5,3 & 1 & 2,9 \\
$\quad$ Dependência grave & & & & &
\end{tabular}

$\mathrm{Na}$ T abela 3, observa-se que $64,7 \%$ dos respondentes considerava sua saúde regular. Comparando sexo e autoaval iação de saúde, os homens autoavaliaram melhor sua saúde do que as mulheres. $33,3 \%$ dos idosos consideraram sua saúde excelente/ muito boa e boa, enquanto que $21 \%$ das mulheres se incluiu nesta categoria.

A morbidade referida de maior prevalência foi a artropatia $(55,9 \%)$, seguida da hipertensão $(52,7 \%)$. A mbas mais frequentes entre as mulheres do que entre os homens. 0 problema de saúde que mais limitava as atividades rotineiras foi a artropatia $(35,3 \%)$. A pesar desta ser mais frequente entre as mulheres, trazia limitação mais intensa para os homens $(33,3 \%)$ do que para as pessoas do sexo feminino $(15,4 \%)$.

Quanto à realização das AVD, destaca-se que $35,3 \%$ dos idosos eram independentes e que 52,9\% possuíam dependência leve. $\mathrm{Na}$ análise por sexo, as mulheres apresentavam maiores percentuais de dependência leve, moder ada e grave $(63,2 \%, 10,5 \%$ e $5,3 \%$ respectivamente) do que os homens $(40,0 \%$, $6,7 \%$ e $0 \%$, respectivamente), que eram mais independentes $(53,3 \%)$.

\section{DISCUSSÃO}

A capacidade funcional da pessoa idosa é influenciada por diversos fatores. E ntre eles estão a 
existência de doenças crônicas, as características socioeconômicas e demográficas, a autoavaliação de saúde e a realização de atividades sociais e em comunidade ${ }^{(2,3,11)}$.

A distribuição por sexo da população idosa da comunidade estudada foi um achado inesperado, pois a literatura mostra uma predominância masculina na ár ea rural(5,12). E sta estaria relacionada à migração das mulheres para as cidades, buscando atividades menos árduas, al ém de mudanças residenciais, buscando a proximidade dos filhos, moradores de áreas urbanas ${ }^{(4,6,12)}$. É possível que essa diferença ocorra por ser mais frequente a migração masculina na área estudada e pelo el evado percentual de idosas solteiras que residem na comunidade. A faixa etária predominante foi de 60 a 69 anos, caracterizando uma população idosa jovem. Esse dado assemelha-se ao encontrado em outros estudos de comunidades urbanas brasileiras $^{(13,14)}$ e rurais do estado(6).

A baixa escolaridade encontrada converge com os resultados de estudos rurais, em que a maior parte dos participantes possuía até quatro anos de estudo(5,6) e reflete 0 pouco acesso à educação no passado. Verificou-se maior escolaridade feminina que masculina, diferente do ocorrido em outros estudos urbanos e rurais, os quais indicam menor escolaridade feminina que a masculina $a^{(5,12,14)}$. É possível que, nessa comunidade, a força física para o trabal ho rural seja mais valorizada que a escolaridade, desestimulando o estudo entre os homens. Sabe-se também que a escolaridade influencia a capacidade funcional, como constatado em estudo urbano, a qual prevaleceu, entre os idosos analfabetos, a dependência moderada e grave ${ }^{(3)}$.

Com relação ao estado conjugal, a maioria dos respondentes possuía companheiro(a), semel hante ao encontrado em estudo da área rural ${ }^{(15)}$. Houve maior proporção de idosos casados do que de idosas, o que pode ser explicado pela mortalidade diferencial por sexo e menor frequência de recasamento feminino(12).

A situação ocupacional predominante foi de aposentados que seguiam trabalhando, principalmente entre os homens. 0 trabalho foi relacionado ao bem-estar entre os octagenários de um município do interior do Estado(16). Parece que, em comunidades rurais, os idosos podem manter a atividade laboral mais facilmente, o que seria inviável em comunidades urbanas, onde prevalecem atividades no setor secundário e terciário, com valorização da produtividade.
Domicílios multigeracionais predominaram no presente estudo, assim como ocorreu em estudo realizado com octagenários em área rural do E stado, em que a maior parte dos idosos vivia com familiares ${ }^{(4)}$. A parcela de domicílios multigeracionais foi superior a de estudos urbanos ${ }^{(14,15,17)}$. Parece ser característico de comunidades rurais de descendência italiana a permanência de um dos filhos com seus pais, a fim de prover apoio, quando necessário.

Os respondentes participavam ativamente das atividades sociais realizadas na comunidade. A participação social foi mais frequente entre os homens e houve maior adesão às atividades desenvolvidas na própria comunidade. Acredita-se que estes achados estejam relacionados à facilidade de acesso e à adequação das atividades ao contexto de vida dos idosos. Julgando a importância da manutenção da capacidade funcional, estudos indicam que a interação social reduz a probabilidade de declínio funcional e de mortalidade ${ }^{(18)}$ e aumenta as chances de dependência entre idosos que não interagem ${ }^{(3)}$.

A pesar da baixa escolaridade, nenhum idoso apresentou comprometimento cognitivo. H ouve relação direta entre escolaridade e escore no M EE M , resultado também demonstrado em outros estudos ${ }^{(10)}$. Os escores obtidos pelos sujeitos foram superiores aos encontrados em outras investigações ${ }^{(4)}$. T rata-se de um aspecto positivo, pois o desempenho cognitivo está relacionado à capacidade dos idosos para realizar as AVD $s^{(19)}$.

A percepção de saúde regular predominou entre os respondentes, assemel hando-se a resultados obtidos em área urbana ${ }^{(14)}$. A percepção negativa foi mais comum entre as mulheres, como ocorreu com octagenários residentes em área rural(4). Este achado pode configurar um fator de risco para as mulheres, por aumentar a probabilidade de declínio funcional e mortalidade ${ }^{(3,18)}$.

E $m$ relação às morbidades, a mais frequente e causadora de maior limitação foi a artropatia, seguida da hipertensão arterial. A maior prevalência de morbidades crônicas entre as mulheres corrobora com os achados de outros estudos ${ }^{(14)}$. Sabe-se que as doenças crônicas, de um modo geral, prejudicam a realização das AVDs entre os ido$\mathrm{sos}^{(2)}$. E $\mathrm{m}$ pesquisa na área rural e na presente investigação, a artropatia foi a doença que mais prejudicou a realização das atividades diárias ${ }^{(4)}$. T al morbidade também está associada com dependência nas AVD em estudo desenvolvido em área urba- 
no (2). 0 cotidiano de atividades árduas no campo pode ag ravar os danos provocados pela artropatia.

H ouve um percentual el evado de idosos com dependência leve se comparado aos resultados de estudo realizado em uma comunidade rural da Itália, no qual predominou a independência ${ }^{(11)}$. E ntretanto, outros fatores podem estar influenciando a diferença entre esses resultados tais como: as condições de vida e de trabal ho desses idosos, que cumulativamente prejudicam o sistema osteomuscular, dificultando a real ização das AVD s.

Verificou-se maior prejuízo na realização das AVD entre as mulheres em relação aos homens, resultado compartilhado por outros estudos ${ }^{(3,14)}$. 0 sexo feminino foi relacionado à dependência funcional em estudo realizado em área urbana ${ }^{(3)}$. 0 maior grau de dependência entre as mulheres pode ser responsável por sua pior percepção de saúde e menor participação social.

\section{CONCLUSÕES}

0 estudo obteve 0 alcance de seus objetivos e contribuiu para a caracterização dos idosos que vivem no meio rural do Brasil, já que ainda são poucos os estudos realizados nesse meio. A metodologia e o instrumento de coleta foram adequados em relação aos objetivos propostos. A principal limitação do estudo foi o pequeno número de participantes, que impossibilitou a generalização dos achados para a população geral, mas promoveu a car acterização das condições de vida dos idosos que residem na comunidade onde 0 estudo foi desenvolvido.

Os idosos da comunidade estudada eram, em sua maioria, do sexo feminino, idosos jovens e de baixa escolaridade. Preponderou o arranjo domiciliar multigeracional, que foi mais frequente entre as mulheres. A maioria dos idosos afirmou ser aposentada e continuar trabalhando, contribuindo, assim, duplamente para a renda familiar. A participação nas atividades sociais da comunidade foi frequente, o que pode apontar para a existência de uma rede de apoio social ao idoso, a qual 0 acolhe, val oriza e o insere socialmente ${ }^{(20)}$.

M esmo possuindo baixa escolaridade, 0 desempenho no MEEM foi adequado para o ponto de corte estabelecido, não sendo demonstrado comprometimento cognitivo na época da entrevista. As difer enças entre os escor es femininos e masculinos foram mínimas ${ }^{(20)}$.
Predominaram a autoavaliação de saúde regular e a percepção negativa entre as mulheres. Houve maior preval ência de morbidades entre as mulheres. A artropatia foi a morbidade mais citada e a maior causadora de limitações nas AVD ${ }^{(20)}$.

A dependência leve ocorreu em uma parcela significativa da população, sinalizando para um estado inicial de declínio funcional. Os graus de dependência mais severos foram encontrados principalmente entre as mulheres ${ }^{(20)}$.

$\mathrm{N}$ ova Roma do Sul se encontra em estágio de franco envelhecimento populacional. Os resultados refer entes à capacidade funcional indicam probabilidade aumentada de declínio funcional entre os idosos da comunidade estudada(20).

São necessárias ações em saúde para a prevenção dos danos ocupacionais resultantes do trabal ho árduo realizado ao longo da vida, tratamento de morbidades e reabilitação dos idosos dependentes, resultando em um envel hecimento ativo e com qualidade de vida. Estas ações devem ser individualizadas e adequadas ao contexto em que vivem as pessoas idosas.

$N$ esse sentido, a enfermagem possui papel central, pois é de sua prática profissional organizar e executar atividades individuais e col etivas de promoção da saúde e de prevenção de morbidades, especialmente no âmbito da A tenção Básica. A assistência centrada na integralidade facilita a abordagem da família tor nando efetivas as orientações dispensadas aos cuidadores de idosos dependentes.

Sugere-se a real ização de estudos longitudinais com idosos de áreas rurais com o intuito de melhor compreender a evolução da capacidade funcional de pessoas moradoras nessas áreas.

\section{REFERÊ NCIAS}

1 Palloni A, Pelláez M. H istórico e natureza do estudo. In: L ebrão M L, D uarte YAO. SA BE: Saúde, Bem-estar e Envelhecimento: o projeto SABE no município de São Paulo: uma abordagem inicial. Brasília (DF): O rganização Pan-A mericana da Saúde; 2003. p. 1532.

2 Alves LC, L eimann BCQ, Vasconcelos M EL, CarvaIho M S, Vasconcelos AG G, Fonseca T CO, et al. A influência das doenças crônicas na capacidade funcional dos idosos do município de São Paulo. Cad Saúde Pública. 2007;23(8):1924-30. 
3 RosaT E C, Benício M HD, Larorre M RD 0, Ramos LR. $F$ atores deter minantes da capacidade funcional entre idosos. Rev Saúde Pública. 2003;37(1):40-8.

4 M orais EP, Rodrigues RQP, G erhardt TE. Os idosos mais vel hos no meio rural: realidade de vida e saúde de uma população do interior gaúcho. Texto Contexto Enferm. 2008;17(2):374-83.

5 Silva J. 0 idoso do município de A rambaré - RS: um contexto rural de envelhecimento [ dissertação] . Porto Alegre: Escola de Enfermagem, U niversidade F ederal do Rio G rande do Sul; 2005.

6 A Icântara LR. I dosos rurais: fatores que influenciam trajetórias e acesso a serviços de saúde no município de Santana da Boa Vista/ RS [ dissertação] . Porto Alegre: Escola de E nfer magem, U niversidade F ederal do Rio G rande do Sul; 2009.

7 Ramos LR, Rosa TEC, Oliveira ZM ., M edina M CG, Santos F R G. Perfil do idoso em área metropolitana na região sudeste do Brasil: resultados de inquérito domiciliar. Rev Saúde Pública. 1993;27(2):87-94.

8 Instituto Brasileiro de G eografia e E statística, Diretoria de Pesquisas, Departamento de População e I ndicadores Sociais. Censos [ Internet] . R io de J aneiro; 2003 [ citado 2007 out 16] . Disponível em: http:/ / www.ibge.gov.br.

9 I caza M C, A bala C, Projeto SA BE. M inimental State Examination (M M SE ) del estudio de demencia en Chile: análisis estatístico. W ashington: OPA S; 1999.

10 Bertolucci PHF, Brucki SM, Campacci SR, Juliano Y. 0 mini-exame do estado mental em uma população em geral: impacto da escolaridade. Arq N euro-Psiquiatr. 1994;52(1):1-17.

11 Rozzini R, Frisoni GB, Ferrucci L, Barbisoni $P$, Bertozzi B, Trabucchi $M$. The effect of chronic diseases on physical function. Comparison between activities of daily living scales and the Phy- sical Performance Test. Age Ageing. 1997;26(4): 281-7.

12 Camarano AA, Kanso S, L eitão e M ello J. Como vive o idoso brasileiro? In: Camarano AA, organizador. Os novos idosos brasileiros: muito além dos 60 ? Rio de Janeiro: IPEA; 2004. p. 25-73.

13 Braga C, L autert L. Caracterização sociodemográfica dos idosos de uma comunidade de Porto A legre, Brasil. Rev G aúcha Enferm. 2004;25(1):44-55.

14 Feliciano AB, M oraes AS, F reitas ICM . 0 perfil do idoso de baixa renda no município de São Carlos, São Paulo, Brasil: um estudo epidemiológico. Cad Saúde Pública. 2004;20(6):1575-85.

$15 \mathrm{G}$ ama E V, D amian J, D el M olino J P, L opez M , Perez $M$, I glesias F. A ssociation of individual activities of daily living with self related health in older people. Age Ageing. 2000;29(3):267-70.

$16 \mathrm{X}$ avier F M F, Ferraz M P, M arc N, Escosteguy NU, $M$ origuchi EH. E Iderly people's definition of quality of life. Rev Bras Psiquiatr. 2003;25(1):31-9.

17 Paskulin LM G, Vianna LAC. Perfil sociodemog ráfico e condições desaúde auto-r eferidas de idosos de Porto Alegre. Rev Saúde Pública. 2007;41(5):757-68.

$18 \mathrm{~L} \mathrm{eeY}$. T he predictive value of self assessed general physical and mental health on functional decline and mortality in older adults. J Epidemiol Community $H$ ealth. 2000;54(2):123-9.

19 Castro KCM , Guerra RO. Impact of cognitive performance on the functional capacity of an elderly population in $\mathrm{N}$ atal, Brazil. Arq N euro-P siquiatr. 2008;66(4):809-13.

20 Rigo II. Avaliação da capacidade funcional dos idosos de uma comunidade rural do R io G rande do Sul [ monografia] . Porto A legre: Escola de E nfermagem, U niversidade F ederal do R io G rande do Sul; 2008.

\section{Endereço da autora / Dirección del autor / Author's address:}

Ilva I nês Rigo

Av. Independência, 50, ap. 406, Independência

90035-070, Porto Alegre, RS

E-mail: riguetta@yahoo.com.br
Recebido em: 12/ 10/ 2009

A provado em: 07/ 05/ 2010 